\title{
Arginine and citrulline protect intestinal cell monolayer tight junctions from hypoxia-induced injury in piglets
}

\author{
John C. Chapman', Yuying Liư', Limin Zhu² and J. Marc Rhoads²
}

BACKGROUND: Arginine (Arg) is deficient in the serum of the preterm neonate and is lower in those developing intestinal ischemia. We investigated whether Arg or its precursor, citrulline (Cit), protects intestinal tight junctions (TJs) from hypoxia (HX) and determined whether inducible nitric oxide (NO) plays a role.

METHODS: Neonatal piglet jejunal IPEC-J2 cell monolayers were treated with Arg or Cit, reversible and irreversible NO synthetase (NOS) inhibitors, and were exposed to HX. TJs were assessed by serial measurements of transepithelial electrical resistance (TEER), flux of inulin-fluorescein isothiocyanate, and immunofluorescent staining of TJ proteins.

RESULTS: We found that Arg and Cit were protective against HX-related damage. At the final time point (14h), the mean TEER ratio (TEER as compared with baseline) for Arg + HX and Cit + $\mathrm{HX}$ was significantly higher than that for HX alone. Both Arg and Cit were associated with decreased inulin flux across hypoxic monolayers and qualitatively preserved TJ proteins. Irreversible inhibition of NOS blocked this protective effect. Lipid peroxidation assay showed that our model did not produce oxidant injury.

CONCLUSION: Arg and Cit, via a mechanism dependent on NO donation, protected intestinal epithelial integrity.

is testinal injury and inflammation resulting from ischemia is integral to the pathogenesis of multiple disease states affecting diverse populations from necrotizing enterocolitis (NEC), spontaneous perforation, and hypoxic injury in the context of cardiac or lung disease in infants, to atherosclerotic mesenteric ischemia and hypoxic injury related to venous thrombosis, autoimmune disease, or chronic cardiopulmonary disease in adult and geriatric populations. NEC, as one example, is the most common gastrointestinal disorder in low birth weight infants, and the total annual cost of caring for affected infants in the United States is estimated to be around $\$ 5$ billion $(1,2)$. NEC has been described as an aberrant reaction of the immature intestinal immune system that occurs in the context of enteral nutrition and is associated with mucosal injury, barrier compromise, and systemic immune response $(3,4)$.

Nitric oxide (NO) production by inducible NO synthetase (iNOS) increases in the presence of acute intestinal injury
(5-7). However, there are conflicting data about the effect of this increase. Excessive production of NO can be destructive to intestinal tissues after ischemia/reperfusion injury (8). Conversely, it has been observed that NO and NOS activation do not have deleterious effects on epithelial barrier function (9). In addition, inhibition of iNOS has been shown to exacerbate inflammation during acute intestinal injury and to delay repair (10).

Arginine (Arg) is the physiological substrate for NO synthesis and has been recognized as an enhancer of protein synthesis and wound healing in vivo (11). Arg is deficient in preterm neonates due to inadequate availability in the diet and the underdevelopment of its synthetic pathways in the small intestine (12). Serum levels of Arg have been shown to be low in patients with NEC, drifting downward $1 \mathrm{wk}$ before the onset of NEC, suggesting that Arg may be essential to a process that is protective against NEC $(13,14)$. Arg has been shown to stimulate intestinal cell migration and recovery of intestinal monolayer transepithelial resistance in a NO-dependent manner (15-17). Arg is synthesized from citrulline (Cit) by the sequential action of the cytosolic enzymes argininosuccinate synthetase and argininosuccinate lyase. Cit is potentially a key precursor of Arg that can then serve as a substrate for the production of $\mathrm{NO}$ and polyamines. Oral supplementation of L-Cit has been shown to increase plasma L-Arg concentration and augment NO-dependent signaling in a dose-dependent manner (18). In addition, similar to Arg, serum levels of Cit have also been shown to be low in premature infants and so may play a role in the pathophysiology of NEC (19). We hypothesized that either Arg or Cit may protect intestinal monolayers from hypoxia (HX)-mediated damage and that the NO synthetic pathway may be involved.

\section{RESULTS}

Transepithelial electrical resistance (TEER) was maintained in IPEC-J2 monolayers treated with Arg and Cit. IPEC-J2 cell monolayers were exposed to HX, TEER measurements were made hourly with brief reoxygenation for $10 \mathrm{~min}$, and the ratio of each value to the value of the resistance before beginning $\mathrm{HX}$ was determined. Monolayers that were not treated and were not exposed to HX were measured as controls. TEER for these monolayers remained constant throughout the period of

'Department of Pediatrics, Division of Neonatology, University of Texas Health Science Center, Houston, Texas; ${ }^{2}$ Department of Pediatrics, Division of Gastroenterology, Pediatric Research Institute, University of Texas Health Science Center, Houston, Texas. Correspondence: John Chapman (John.c.chapman@uth.tmc.edu) 
HX exposure. For monolayers that were exposed to HX but untreated, TEER began to fall, on average, after hour 12 and continued to fall through the period of exposure (Figure 1a). Monolayers treated with Arg and Cit but not exposed to HX were not found to be significantly different from controls at any time point.

TEER measured across the monolayers treated with Arg and Cit and subsequently exposed to HX remained constant throughout the period of HX and were not significantly different from controls at any time point (Figure 1b). HX monolayers treated with Arg or Cit appeared to have a higher TEER as compared with control membranes, but these values did not significantly differ from controls until 13 and $14 \mathrm{~h}$ post-HX. The small increase in TEER may be related to cell swelling, because Arg is transported by systems $\mathrm{y}^{+}, \mathrm{L}$, and $\mathrm{B}^{0 /+}$ (standard terminologies for amino acid exchange systems). The $\mathrm{B}^{0 /+}$ system uptake is coupled to $\mathrm{Na}$ ions; therefore, cells could swell from both Arg and $\mathrm{Na}^{+}$uptake. At the final time point, the mean TEER (expressed as a multiple of baseline TEER) for Arg + HX was 1.25 and the mean TEER for Cit + HX was 1.22 , as compared with 0.14 with HX alone; the mean difference between Arg + HX and HX alone was 1.1, 95\% confidence interval $=0.21-1.9, P<0.001$; Cit $+\mathrm{HX}$ mean difference was 1.08 with $95 \%$ confidence interval $=0.18-1.9, P<0.001$, Figure $1 \mathbf{b}$ ). Note that all experiments in Figure $1 \mathbf{a}, \mathbf{b}$ were done simultaneously. These findings demonstrated that prolonged HX promotes membrane breakdown after about $12 \mathrm{~h}$, which is completely prevented by incubation of Arg or Cit.

TEER was not maintained in IPEC-J2 monolayers treated with Arg or Cit plus the irreversible NOS inhibitor L-N6-(1iminoethyl) lysine (L-NIL). NO formation was subsequently inhibited by the addition of L-NIL, an irreversible inhibitor that is selective to iNOS. TEER was measured in monolayers treated with L-NIL throughout the exposure period and remained steady, indicating that L-NIL was not toxic. For the

a

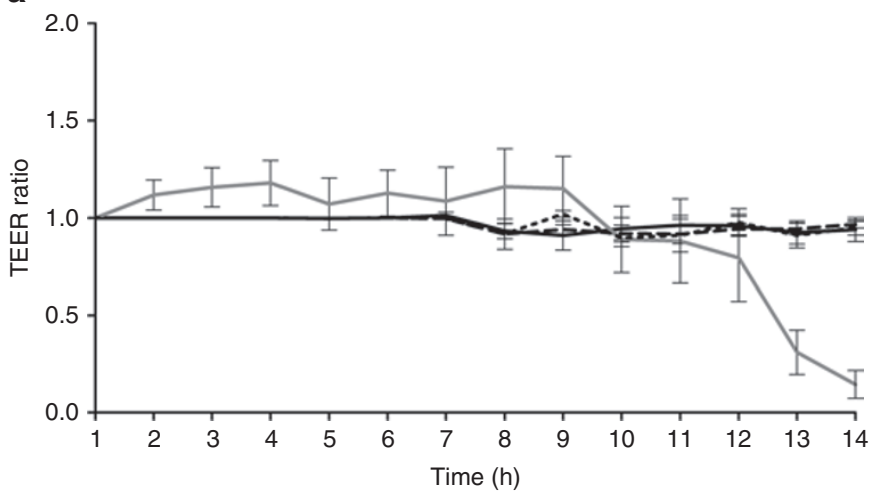

first $9 \mathrm{~h}$, TEER was $20-30 \%$ lower than in controls in monolayers exposed to L-NIL ( $P=$ not significant) At $11 \mathrm{~h}$, TEER rose rapidly, then plummeted as membrane integrity was breached at 13-14 h (Figure 2a). Membrane integrity dropped after $12 \mathrm{~h}$ in all monolayers exposed to L-NIL (with or without Arg or Cit) (Figure 2a). Note that all experiments in Figures 2a,b were done simultaneously; the HX control values are shown in both figures for comparison with Arg and Cit without or with HX. Our findings indicate that inducible NO is required for the beneficial effects of Arg or Cit.

TEER was maintained in IPEC-J2 monolayers treated with Arg or Cit and the reversible iNOS inhibitor NG-Nitro-Larginine (L-NAME). NO formation was inhibited reversibly by the addition of L-NAME, a nonselective and reversible inhibitor of NOS. TEER was measured in monolayers treated with L-NAME throughout the exposure period and was reduced as compared with controls by the final time point (Figure 2b). Arg or Cit was added in concentrations sufficient to reverse the inhibition of NOS. The $12 \mathrm{mmol} / \mathrm{l}$ concentration was chosen based on prior observations that three times the concentration of Arg (as compared with L-NAME) was required for reversal of inhibition (20). Monolayers showed a comparable value of TEER to that of controls at all time points. We conclude that when Arg or Cit was added in sufficient concentrations to reverse the inhibition by L-NAME, TEER was preserved.

Inulin flux was reduced across hypoxic IPEC-J2 monolayers treated with Arg or Cit or with reversible NOS inhibitors. The concentration of inulin crossing the monolayers was measured by fluorescence from samples taken from the supernatant $2 \mathrm{~h}$ after reduction in TEER. Fluorescence readings of labeled inulin flux across the monolayers for which reduction of TEER was similar to untreated controls confirmed barrier compromise for those treated with L-NAME, L-NIL, Arg + L-NIL, or Cit + L-NIL (Figure 3). Fluorescence readings from the

b

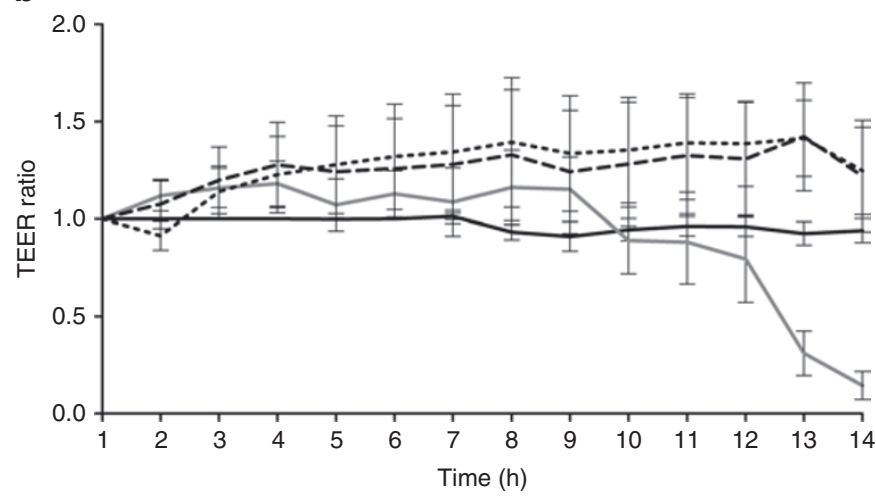

Figure 1. Transepithelial electrical resistance (TEER) of IPEC-J2 monolayers during exposure to hypoxia (HX). (a) TEER of control monolayers not exposed to HX (solid black line) remained constant $(n=8)$. TEER of monolayers exposed to HX but untreated (solid dark gray line) began to fall after hour 12 ( $n=$ 12). TEER of monolayers not exposed to HX and treated with arginine (Arg) (short-dashed line) ( $n=8$ ) or citrulline (Cit) (long-dashed line) ( $n=8)$ were not significantly different from controls at any time point. (b) TEER of monolayers exposed to HX and treated with Arg (short-dashed line) ( $n=16)$ or Cit (longdashed line) $(n=16)$ remained constant and were not significantly different from controls (monolayers not exposed to HX, solid black line; monolayers exposed to $\mathrm{HX}$ but untreated, solid dark gray line) at any time point, with a mean difference at the final time point for Arg $+\mathrm{HX}$ of 1.25 and Cit $+\mathrm{HX}$ of 1.22 , as compared with 0.14 with $\mathrm{HX}$ alone. The mean difference between $\mathrm{Arg}+\mathrm{HX}$ and $\mathrm{HX}$ alone was $1.1,95 \%$ confidence interval $(\mathrm{Cl})=0.21-1.9, P<$ 0.001; Cit $+\mathrm{HX}$ mean difference was 1.08 with $95 \% \mathrm{Cl}=0.18-1.9, P<0.001$. 
a

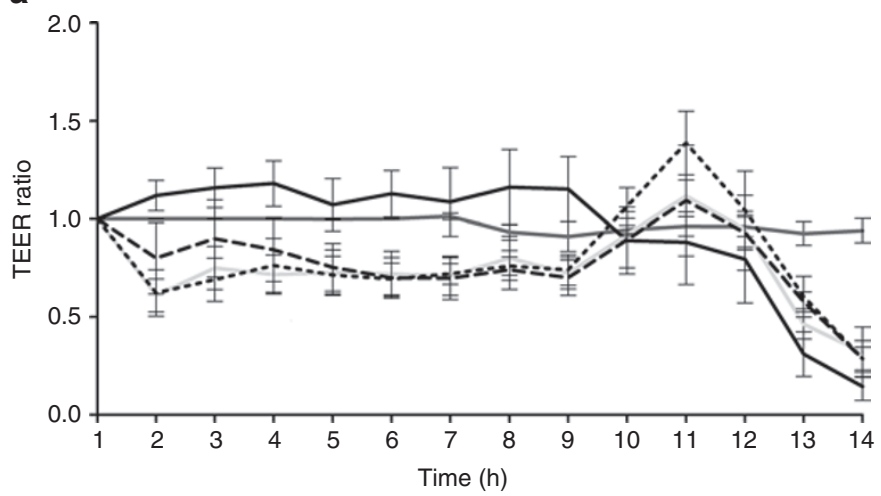

b

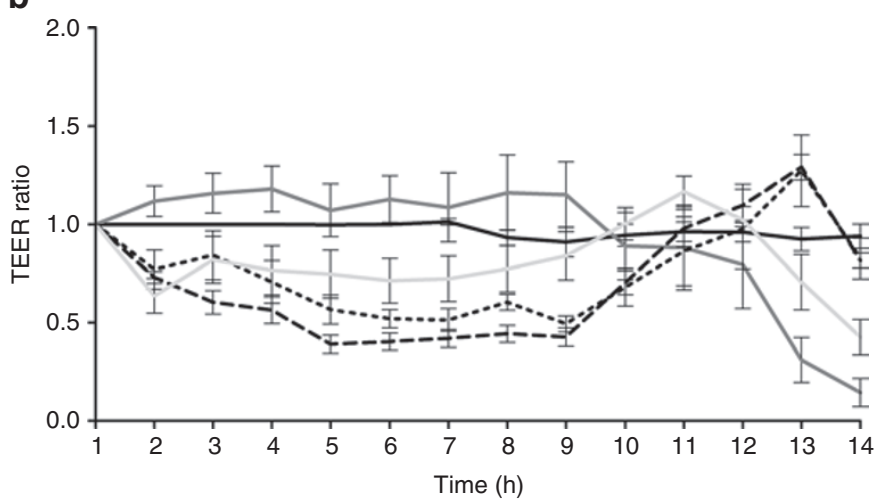

Figure 2. Transepithelial electrical resistance (TEER) of IPEC-J2 monolayers treated with nitric oxide synthetase (NOS) inhibitors during exposure to hypoxia (HX). (a) TEER was reduced in monolayers treated with the irreversible inducible NOS inhibitor L-N6-(1-iminoethyl) lysine (L-NIL) (solid light gray line) as compared with controls (monolayers not exposed to HX, solid black line; monolayers exposed to HX but untreated, solid dark gray line) by the final time point $(n=6)$. TEER also dropped for monolayers treated with arginine (Arg) plus L-NIL (short-dashed line) $(n=6)$ or citrulline (Cit) plus L-NIL (long-dashed line) $(n=6)$ by the final time point. (b) TEER was reduced in monolayers treated with NG-Nitro-L-arginine (L-NAME) (solid light gray line) as compared with controls by the final time point $(n=12)$. TEER was preserved for monolayers treated with Arg (short-dashed line) ( $n=12)$ or Cit (longdashed line) $(n=12)$ in sufficient concentration to override NOS inhibition by L-NAME and was similar to that of controls, with mean difference at final time point between Arg + L-NAME and $\mathrm{HX}$ alone of $0.68,95 \%$ confidence interval $(\mathrm{Cl})=-0.49$ to $1.9, P>0.05$; and Cit + L-NAME mean difference of 0.67 with $95 \% \mathrm{Cl}=-0.5$ to $1.8, P>0.05$.

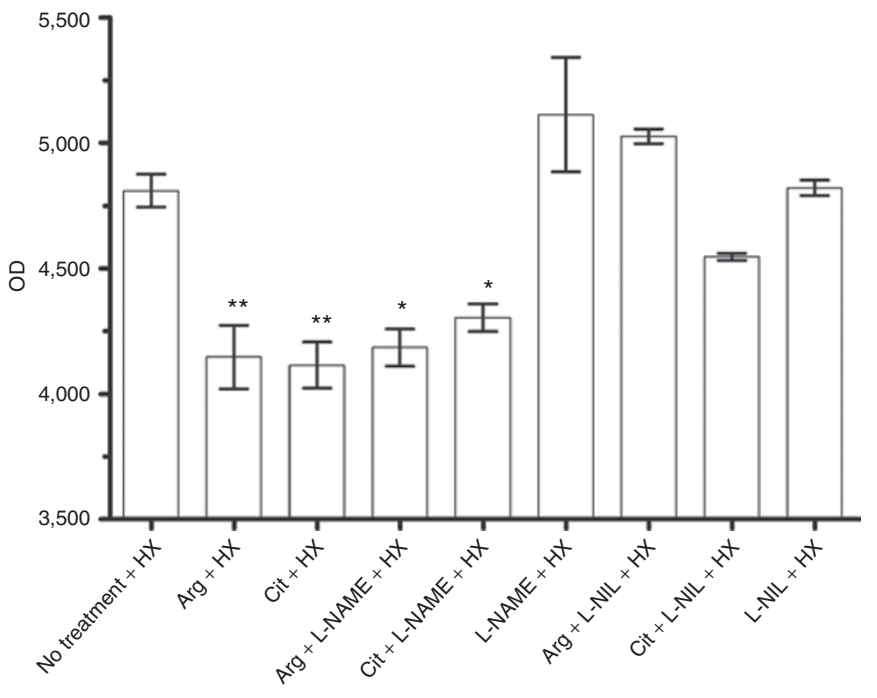

Figure 3. Inulin flux across monolayers. In the setting of hypoxia with L-NIL treatment (with or without arginine (Arg) or citrulline (Cit)), inulin flux as measured by optical density (OD) was increased across the monolayers with nonpreservation of transepithelial electrical resistance (TEER) similar to untreated hypoxic controls, confirming barrier compromise. Inulin flux remained low across the monolayers for which there was no reduction in TEER as compared with controls, that is, hypoxic monolayers treated with Arg, Cit, Arg overriding NG-Nitro-L-arginine (L-NAME), or Cit overriding L-NAME ( $n=5$ for all groups; $\left.{ }^{*} P<0.05,{ }^{*} P<0.01\right)$. L-NIL, L-N6-(1-iminoethyl) lysine.

monolayers treated with Arg or Cit (and those treated with Arg or Cit in sufficient concentrations to reverse NOS inhibition by L-NAME) were significantly lower. (Mean differences between no treatment and various treatments were significant with $P<$ 0.05 for Arg + L-NAME and Cit + L-NAME, and $P<0.01$ for Arg and Cit; Figure 3.)

Immunofluorescent staining of the tight junction (TJ) protein zonula occludens (ZO)-1 was qualitatively different between the variously treated and HX-exposed IPEC-J2 monolayers. We performed immunocytochemical staining of the TJ protein ZO-1 at 14-h post-TEER change to determine the distribution of TJ proteins. Results showed that in HX-exposed monolayers, dissolution of ZO-1 from the intracellular borders occurred, with only hazy remnants of normal cell-cell junctions remaining (Figure 4). Arg or Cit prevented these changes. In the presence of L-NIL, Arg or Cit did not protect from TJ damage, whereas in the presence of reversible inhibition with L-NAME, addition of Arg or Cit reversed the damage. These findings identify a HX-induced global impairment of TJ integrity, rather than specific "punctures" in the membrane, a process largely prevented by Arg or Cit supplementation. Similar studies were also performed with an antioccludin antibody, and results were comparable to those with ZO-1, including qualitatively preserved intercellular junctions with Arg or Cit (data not shown but available on request).

\section{Lipid Peroxidation Assay}

Studies were designed to see if the brief exposures to room air during the hourly measurements of TEER were sufficient to allow oxidant damage to the membranes. Thiobarbituric acid reactive substances assay for lipid peroxidation was performed on IPEC-J2 cells subjected to the treatments used in previous studies. This assay also allowed us to determine if there was oxidant damage associated with Arg or Cit, because there are some data to suggest that supplementation of these $\mathrm{NO}$ donors could increase oxidant damage from increased reactive nitrogen species. Our assays showed that there were significant reductions in lipid peroxidation products, comparing the treatment groups ( $\mathrm{HX}$ alone, $\mathrm{HX}+\mathrm{Arg}$, and $\mathrm{HX}+\mathrm{Cit}$ ) with the untreated control group. This finding indicates that Arg or Cit treatment of hypoxic intestinal epithelial cells does not promote oxidation (Figure 5). 

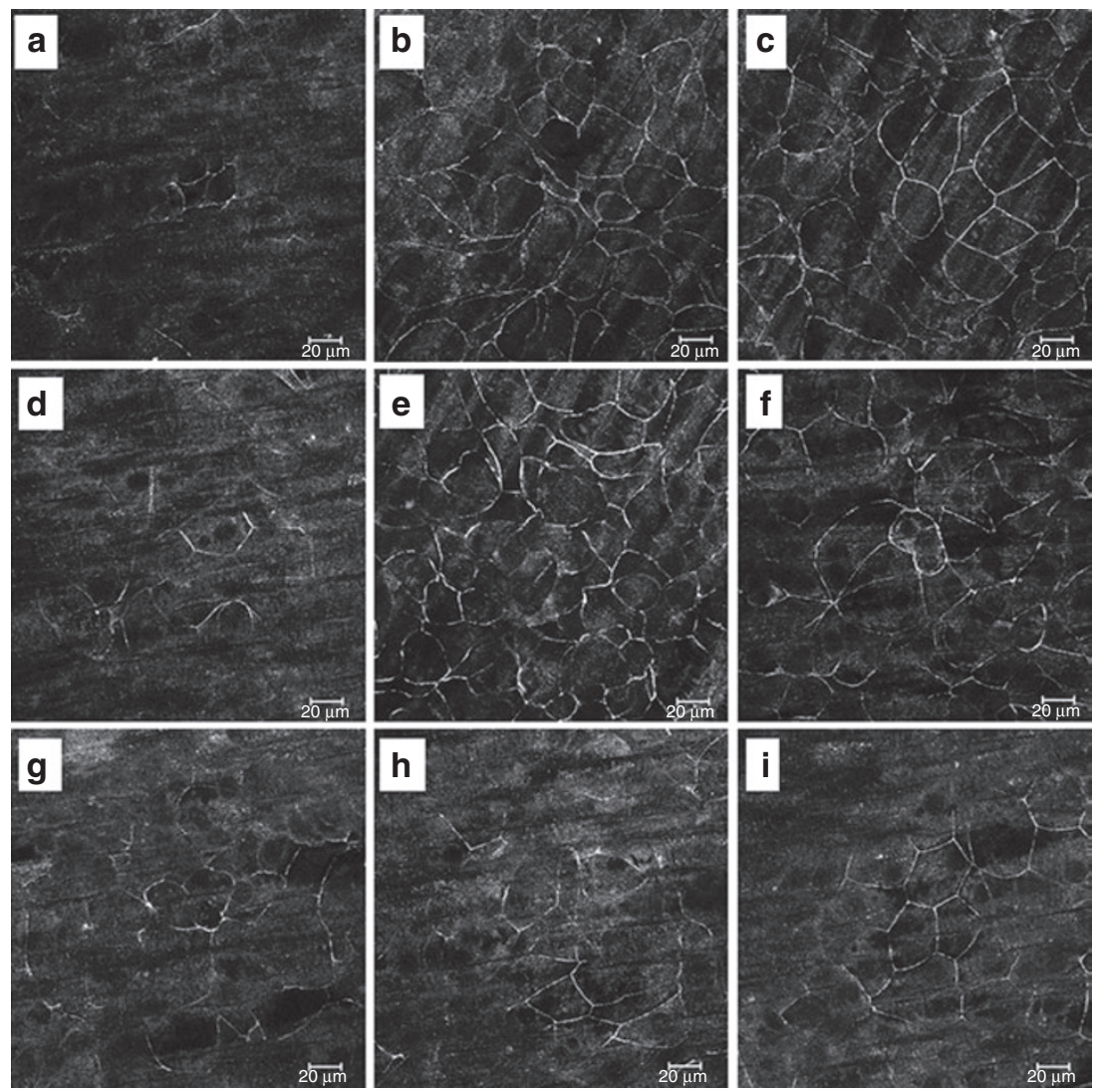

Figure 4. Zonula occludens (ZO)-1 immunofluorescent staining. (a-i) Exposed to hypoxia (HX). (a) HX alone, (b) arginine (Arg), (c) citrulline (Cit), (d) NG-Nitro-L-arginine (L-NAME), (e) Arg + L-NAME, (f) Cit + L-NAME, (g) L-N6-(1-iminoethyl) lysine (L-NIL), (h) Arg + L-NIL, and (i) Cit + L-NIL. In HX-exposed monolayers, dissolution of ZO-1 from the intracellular borders occurred, with only hazy remnants of normal cell-cell junctions remaining. Arg and Cit prevented these changes, but in the presence of L-NIL, supplemental Arg or Cit did not protect from tight junction damage. However, in the presence of reversible inhibition with L-NAME, addition of Arg or Cit in sufficient quantities reversed the effect. Images taken with 400x original magnification.

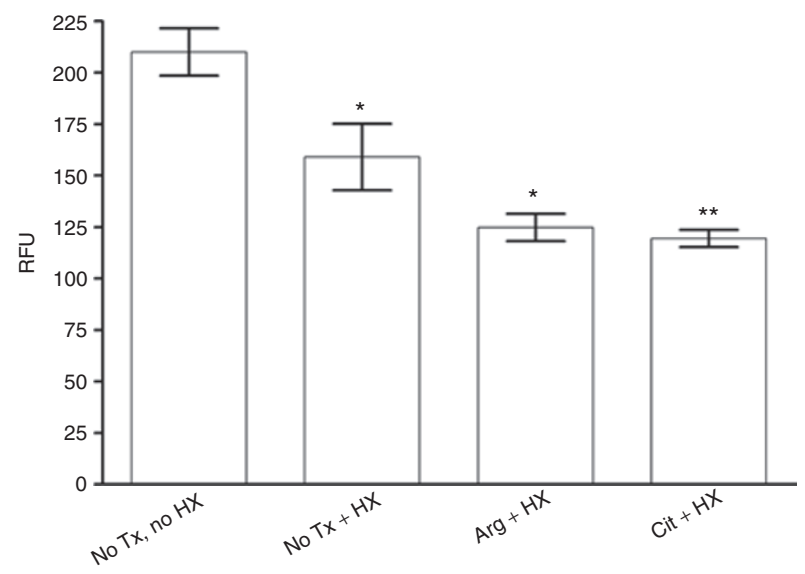

Figure 5. Lipid peroxidation assay. There were significant reductions in lipid peroxidation products between treatment groups (hypoxia $(\mathrm{HX})$ alone, $\mathrm{HX}+$ arginine (Arg), and $\mathrm{HX}+$ citrulline (Cit)) and the untreated (no Tx) control group. Results demonstrate that Arg or Cit treatment does not promote oxidation ( $n=6$ for all groups; ${ }^{*} P<0.05$, ${ }^{* *} P<0.01$ ). Tx, treatment.

\section{DISCUSSION}

With the use of an in vitro model of the intestinal epithelial barrier, this study was able to determine that supplementation of Arg or Cit confers a protective effect when the epithelial cells are subjected to the stress of HX. The IPEC-J2 cell is a primary cell line derived from jejunal epithelium isolated from unsuckled, neonatal piglets. Previous studies have characterized this cell line and reported it to be suitable as an in vitro intestinal model to study the permeability of some antiviral agents $(21,22)$. IPEC-J2 cells are enterocyte-like, produce glycocalyxbound mucin, form microvilli and TJs, and create a functional cell monolayer in vitro (23). In addition, IPEC-J2 cells have been used in numerous studies of Gram-negative bacteria/host interactions (24,25), and infection or Escherichia coli lipopolysaccharide induces appropriate cytokine responses in these cells $(21,26)$. Our results, along with the aforementioned reports from the literature, clearly indicate the potential of IPEC-J2 cell monolayers as a model for intestinal permeability studies.

The HX produced by this model, which includes a prolonged exposure to hypoxic conditions, is probably more severe and prolonged than hypoxic injuries encountered in vivo; however, the model does not include leukocytes or lamina propria elements that might magnify the injury, e.g., by releasing reactive oxidant species or additional NO. The time required for HX to compromise barrier integrity by interrupting TJs in in vitro polarized cell monolayers appears to be different among the cell types. For most of the intestinal epithelial cells-e.g., Caco-2 and IEC-6-decreases in TEER were observed $\sim 6 \mathrm{~h}$ after HX 
(27). However, we observed a roughly twofold increase in the time before decrease in TEER; this may be related to a prior observation that cells in IPEC-J2 monolayers form tighter intercellular junctions than those in Caco-2 monolayers (22). The inclusion of NOS inhibitors and the subsequent results allow us to conclude that this protective effect is dependent on the production of NO and dependent on iNOS-derived NO.

Conflicting data exist regarding the effect of NO on the intestinal epithelium. Studies examining chronic intestinal inflammation have shown that NO exacerbates intestinal injury through nitrotyrosylation (28). Our model, which exposes the intestinal epithelium to more acute injury, involves no increase in oxidative stress associated with supplementation of $\mathrm{NO}$ donors. Other studies of acute intestinal damage have shown that iNOS-derived NO can have beneficial effects, but the exact mechanisms are just now being explored $(10,29)$. One possible mechanism is $\mathrm{HX}$-inducible factor-1 induction. HX-inducible factor-1 is the master regulator of cellular response to hypoxia. Intracellular $\mathrm{HX}$-inducible factor- 1 is stabilized in response to NO donors, independent of cyclic guanosine monophosphate and dependent on S-nitrosylation (30). One might speculate that under hypoxic conditions, intestinal cellular monolayers undergo activation of HX-inducible factor-1 that is optimally preserved over a prolonged time course by NO donors such as Arg or Cit. What is known is that constitutive iNOS activity is present in the intestinal epithelia of several species. Furthermore, upregulation of the transcription and translation of iNOS has been shown to occur within 30 min of injury in multiple models. This ability to rapidly increase NO synthesis, in a tissue that is vulnerable to injury, suggested a beneficial role for NO in barrier maintenance.

The iNOS-dependent protective effect of the NO donors, Arg and Cit, demonstrated by our study, is consistent with that reported in several other studies. NO in the context of Arg supplementation has been shown to be involved in cell migration in razor-injured porcine intestinal epithelial cell monolayers (16). iNOS-derived NO following Arg supplementation has also been shown to be involved in re-epithelialization of laser-wounded renal tubular cell monolayers and deoxycholate-injured porcine ileal mucosa mounted in Ussing chambers $(15,31)$.

The barrier function of the intestinal mucosa is particularly important for individuals such as premature infants, who are largely dependent on innate immunity. The compromise of this physical barrier is recognized as a key component of the pathophysiology of NEC and intestinal injury in the context of congenital heart disease and chronic lung disease. Arg supplementation prevents and accelerates recovery from ischemic intestinal damage in animal models (32-34). Supplementation of Arg for the prevention of NEC in humans has also been examined, and in two studies, Arg supplementation reduced the incidence of NEC with either enteral or parenteral supplementation $(35,36)$.

We have presented evidence that NO is involved in the protective effect of Arg and Cit supplementation during intestinal epithelial HX. The roles of polyamines (putrescine, spermidine, and spermine), also products of Arg and Cit metabolism that are known to be protective in the gut, were not examined. In addition, the study design does not take into account the full physiologic context of the epithelium. For example, although the choice of the IPEC-J2 cell line does approximate the in vivo architecture of intestinal epithelia more than transformed cell lines, there is the possibility that lamina propria elements (nerves, resident macrophages, T cells, and dendritic cells) would react to Arg or Cit in such a way as to prevent protection in other models of bowel damage (21). However, this was not the case in bile salt injury to the piglet ileal epithelium or in the human studies cited $(15,35,36)$. Preclinical studies examining the role of Cit feeding are needed to address these issues and others, including establishing an optimal oral dose and mode of delivery for subjects with low levels of Arg or Cit who are at risk of intestinal injury.

To our knowledge, this is the first study to examine whether Cit might also be protective and to suggest that supplementation of Cit for prevention or treatment of ischemic injury of the intestine should be examined. After ingestion, Cit would be converted to Arg by the sequential action of the cytosolic enzymes argininosuccinate synthetase and argininosuccinate lyase, and Cit has been shown to serve as a donor of NO (18). Cit, an important component of watermelon, can be given orally with little risk of causing diarrhea, an occasional side-effect of Arg (37).

\section{METHODS}

\section{Cell Culture and Polarized Monolayer Growth}

The IPEC-J2 cell line was obtained from Douglas G. Burrin, Baylor College of Medicine. This cell line is a nontransformed intestinal cell line that was developed by H.M. Bershneider from jejunal epithelium isolated from a neonatal piglet (23). It has been demonstrated that IPEC-J2 cells, unlike transformed cell lines, form epithelia of enterocyte-like cells with microvilli, TJs, and glycocalyx-bound mucin, and produce an appropriate cytokine response to bacterial pathogens (21). All experiments were carried out in cells between passages 38 and 56. The IPEC-J2 cells were grown in Dulbecco's modified eagle medium with high D-glucose ( $4.5 \mathrm{~g} / \mathrm{l})$ (Invitrogen, Carlsbad, CA) supplemented with $20 \%$ fetal bovine serum, 0.1 million units/l penicillin, and $100 \mathrm{mg} / \mathrm{l}$ streptomycin (Atlanta Bio, Lawrenceville, GA), and maintained in an atmosphere of $5 \% \mathrm{CO}_{2}$ at $37^{\circ} \mathrm{C}$. Cells were transferred to 12 -well collagen-coated transwells with a $0.4 \mu \mathrm{m}$ pore size (Corning Life Sciences, Lowell, MA) and were plated at minimum density of $1 \times 10^{6}$ cells/well. Cell growth was observed for confluence by light microscopy, and TEER was measured by EVOM epithelial voltohmmeters (World Precision Instrument, Sarasota, FL) every 2-3 d. Monolayers were considered confluent when TEER values reached a plateau between 800 and $1,200 \mathrm{Ohm} \times \mathrm{cm}^{2}$.

\section{Monolayer Treatment}

An amount of $4 \mathrm{mmol} / \mathrm{l}$ L-arginine hydrochloride (Arg) (Fisher Scientific, Pittsburgh, PA) or Cit (Sigma, St Louis, MO) was added to Basal Medium Eagle (Sigma) bathing the monolayers overnight in the medium. The Basal Medium Eagle contains $<0.12 \mathrm{mmol} / \mathrm{l}$ Arg. A prior study examining Arg and Cit synthesis in enterocytes isolated from neonatal pig jejuna found that Arg was typically present in concentrations from $0.03 \pm 0.01 \mathrm{mmol} / \mathrm{l}$ at $0 \mathrm{~d}$ of life to $1.53 \pm 0.28 \mathrm{mmol} / \mathrm{l}$ at $7 \mathrm{~d}$ of life. We chose the $4 \mathrm{mmol}$ concentration to ensure that we were modestly above naturally occurring levels, in case of any metabolism or degradation of Arg. This study also showed that Cit served as an effective precursor for Arg synthesis in supplementations ranging from 0.5 to $5 \mathrm{mmol}$ concentrations (38). Another study examining jejunal amino acid concentrations after human test subjects were fed a protein-rich test meal showed an increase in Arg concentration from $0.23 \pm 0.07 \mathrm{mmol} / \mathrm{l}$ before the meal to $2.06 \pm 0.11 \mathrm{mmol} / \mathrm{l}$ after, which is comparable with levels found in the piglet jejuna and similar to the $4 \mathrm{mmol}$ concentration chosen for our study (39). Inhibitors of iNOS including L-NAME and L-NIL (Sigma) were applied to monolayers overnight at a concentration of $12 \mathrm{mmol} / \mathrm{l}$, on the basis of previous 
studies that showed that three times more L-NAME than Arg was required for adequate competitive inhibition. When reversal of inhibition by L-NAME was planned, the ratio was reversed, and $12 \mathrm{mmol} / \mathrm{l}$ of either Arg or Cit was combined with $4 \mathrm{mmol} / \mathrm{l}$ of L-NAME.

\section{Monolayer Exposure to HX and TEER Measurement}

Monolayers cultured in transwells were exposed to $\mathrm{HX}\left(5 \% \mathrm{CO}_{2}\right.$ and $95 \%$ nitrogen) within a modular incubator chamber (BillupsRothenberg, Del Mar, CA). Oxygen concentrations were determined to be $<1 \%$ by Micro IV oxygen sensor (GfG Instrumentation, Ann Arbor, MI). Monolayers were removed from the HX chamber at hourly intervals for measurement of TEER for $5 \mathrm{~min}$. TEER was used as a measure of transepithelial permeability (40). The monolayer barrier was considered compromised when TEER measurements fell below $1 \mathrm{Ohm} \times \mathrm{cm}^{2}$. Although we cannot assume that this exposure to $\mathrm{HX}$ accurately simulates in vivo hypoxic stress, studies using similar techniques, although with the T84 cell line (transformed and colonic cells), demonstrated an effect on TJs and epithelial permeability by measurements of TEER and fluorescein-dextran flux (41).

\section{Inulin Flux Measurement}

Fluorescein isothiocyanate-labeled inulin (Sigma), a polyfructose molecule with molecular weight $\sim 5,000$, was added to the basal well of the transwell system after TEER measurements demonstrated loss of confluence. Supernatants were taken from the apical well $2 \mathrm{~h}$ later and fluorescence was measured by TECAN Infinite M200 fluorimeter (TECAN US, Research Triangle Park, NC). Measurements were reported in relative fluorescence units.

\section{Immunofluorescence Assay}

Monolayers were incubated with rabbit anti-ZO-1 at $4 \mu \mathrm{g} / \mathrm{ml}$ for $12 \mathrm{~h}$ at $4{ }^{\circ} \mathrm{C}$, then washed and incubated with goat anti-rabbit Alexafluor 488 (Life Technologies, Grand Island, NY) at a dilution of 1:200 for $30 \mathrm{~min}$ at room temperature. Monolayers were mounted with Prolong Gold antifade reagent (Life Technologies, Grand Island, NY) with 4',6-diamidino-2-phenylindole. Images were digitally captured using a confocal microscope (Zeiss LSM510; Carl Zeiss Microscopy, Thornwood, NY) equipped with a laser diode $(405 \mathrm{~nm})$ and $\operatorname{argon}(488 \mathrm{~nm})$, helium/neon $(546 \mathrm{~nm})$, and helium/neon $(633 \mathrm{~nm})$ lasers. Antibodies and mounting medium were from Invitrogen.

\section{Lipid Peroxidation Assay}

Thiobarbituric acid reactive substances assay (Cell Biolabs, San Diego, CA) for lipid peroxidation was performed according to the manufacturer's protocol. Colored reaction products were measured by fluorimeter at $540 \mathrm{~nm}$ excitation and $590 \mathrm{~nm}$ emission and reported in relative fluorescence units.

\section{Statistical Analysis}

Statistical analysis was performed with one-way and two-way ANOVA using Prizm 4.0 (GraphPad software, San Diego, CA). Experimental results are expressed as means \pm SE. Dunnett's and Tukey's multiple comparison tests were used for comparison of multiple groups with a control group. $P$ values of $<0.05$ were considered statistically significant.

\section{STATEMENT OF FINANCIAL SUPPORT}

This work was supported by the Department of Pediatrics, Division of Gastroenterology, University of Texas Health Science Center, Houston, Texas. Additional funds were provided by Kathleen Kennedy, director of the Division of Perinatal-Neonatal Medicine, Department of Pediatrics, University of Texas Health Science Center, Houston, Texas, from the Richard W. Mithoff Professor Grant.

Disclosure: The authors declared no conflict of interest.

\section{REFERENCES}

1. Bisquera JA, Cooper TR, Berseth CL. Impact of necrotizing enterocolitis on length of stay and hospital charges in very low birth weight infants. Pediatrics 2002;109:423-28.

2. Henry MC, Moss RL. Necrotizing enterocolitis. Annu Rev Med 2009;60:111-24.
3. Martin CR, Walker WA. Intestinal immune defences and the inflammatory response in necrotising enterocolitis. Semin Fetal Neonatal Med 2006;11:369-77.

4. Morowitz MJ, Poroyko V, Caplan M, Alverdy J, Liu DC. Redefining the role of intestinal microbes in the pathogenesis of necrotizing enterocolitis. Pediatrics 2010;125:777-85.

5. Banan A, Fields JZ, Zhang Y, Keshavarzian A. iNOS upregulation mediates oxidant-induced disruption of F-actin and barrier of intestinal monolayers. Am J Physiol Gastrointest Liver Physiol 2001;280:G1234-46.

6. Kalff JC, Schraut WH, Billiar TR, Simmons RL, Bauer AJ. Role of inducible nitric oxide synthase in postoperative intestinal smooth muscle dysfunction in rodents. Gastroenterology 2000;118:316-27.

7. Miller MJ, Zhang XJ, Sadowska-Krowicka H, et al. Nitric oxide release in response to gut injury. Scand J Gastroenterol 1993;28:149-54.

8. Kozar RA, Holcomb JB, Hassoun HT, Macaitis J, DeSoignie R, Moore FA. Superior mesenteric artery occlusion models shock-induced gut ischemiareperfusion. J Surg Res 2004;116:145-50.

9. Olson N, Greul AK, Hristova M, Bove PF, Kasahara DI, van der Vliet A. Nitric oxide and airway epithelial barrier function: regulation of tight junction proteins and epithelial permeability. Arch Biochem Biophys 2009;484:205-13.

10. McCafferty DM, Mudgett JS, Swain MG, Kubes P. Inducible nitric oxide synthase plays a critical role in resolving intestinal inflammation. Gastroenterology 1997;112:1022-7.

11. Alican I, Kubes P. A critical role for nitric oxide in intestinal barrier function and dysfunction. Am J Physiol 1996;270(2 Pt 1):G225-37.

12. Batshaw ML, Wachtel RC, Thomas GH, Starrett A, Brusilow SW. Arginineresponsive asymptomatic hyperammonemia in the premature infant. J Pediatr 1984;105:86-91.

13. Becker RM, Wu G, Galanko JA, et al. Reduced serum amino acid concentrations in infants with necrotizing enterocolitis. J Pediatr 2000;137:785-93.

14. Zamora SA, Amin HJ, McMillan DD, et al. Plasma L-arginine concentrations in premature infants with necrotizing enterocolitis. J Pediatr 1997;131:226-32.

15. Gookin JL, Rhoads JM, Argenzio RA. Inducible nitric oxide synthase mediates early epithelial repair of porcine ileum. Am J Physiol Gastrointest Liver Physiol 2002;283:G157-68.

16. Rhoads JM, Chen W, Gookin J, et al. Arginine stimulates intestinal cell migration through a focal adhesion kinase dependent mechanism. Gut 2004;53:514-22.

17. Rhoads JM, Liu Y, Niu X, Surendran S, Wu G. Arginine stimulates cdx2-transformed intestinal epithelial cell migration via a mechanism requiring both nitric oxide and phosphorylation of p70 S6 kinase. J Nutr 2008;138:1652-7.

18. Schwedhelm E, Maas R, Freese R, et al. Pharmacokinetic and pharmacodynamic properties of oral $\mathrm{L}$-citrulline and $\mathrm{L}$-arginine: impact on nitric oxide metabolism. Br J Clin Pharmacol 2008;65:51-9.

19. Wu G, Jaeger LA, Bazer FW, Rhoads JM. Arginine deficiency in preterm infants: biochemical mechanisms and nutritional implications. J Nutr Biochem 2004;15:442-51.

20. Yanaka A, Muto H, Fukutomi H, Ito S, Silen W. Role of nitric oxide in restitution of injured guinea pig gastric mucosa in vitro. Am J Physiol 1995;268(6 Pt 1):G933-42.

21. Schierack P, Nordhoff M, Pollmann M, et al. Characterization of a porcine intestinal epithelial cell line for in vitro studies of microbial pathogenesis in swine. Histochem Cell Biol 2006;125:293-305.

22. Pisal DS, Yellepeddi VK, Kumar A, Palakurthi S. Transport of surface engineered polyamidoamine (PAMAM) dendrimers across IPEC-J2 cell monolayers. Drug Deliv 2008;15:515-22.

23. Schierack P, Nordhoff M, Pollmann M, et al. Characterization of a porcine intestinal epithelial cell line for in vitro studies of microbial pathogenesis in swine. Histochem Cell Biol 2006;125:293-305.

24. Skjolaas KA, Burkey TE, Dritz SS, Minton JE. Effects of Salmonella enterica serovars Typhimurium (ST) and Choleraesuis (SC) on chemokine and cytokine expression in swine ileum and jejunal epithelial cells. Vet Immunol Immunopathol 2006;111:199-209.

25. Brown DR, Price LD. Characterization of Salmonella enterica serovar Typhimurium DT104 invasion in an epithelial cell line (IPEC J2) from porcine small intestine. Vet Microbiol 2007;120:328-33. 
26. Liu Y, Fatheree NY, Mangalat N, Rhoads JM. Human-derived probiotic Lactobacillus reuteri strains differentially reduce intestinal inflammation. Am J Physiol Gastrointest Liver Physiol 2010;299:G1087-96.

27. Xia G, Martin AE, Michalsky MP, Besner GE. Heparin-binding EGF-like growth factor preserves crypt cell proliferation and decreases bacterial translocation after intestinal ischemia/reperfusion injury. J Pediatr Surg 2002;37:1081-7; discussion 1081-7.

28. Miller MJ, Thompson JH, Zhang XJ, et al. Role of inducible nitric oxide synthase expression and peroxynitrite formation in guinea pig ileitis. Gastroenterology 1995;109:1475-83.

29. McCafferty DM, Miampamba M, Sihota E, Sharkey KA, Kubes P. Role of inducible nitric oxide synthase in trinitrobenzene sulphonic acid induced colitis in mice. Gut 1999;45:864-73.

30. Sumbayev VV, Budde A, Zhou J, Brüne B. HIF-1 alpha protein as a target for S-nitrosation. FEBS Lett 2003;535:106-12.

31. Noiri E, Peresleni T, Srivastava N, et al. Nitric oxide is necessary for a switch from stationary to locomoting phenotype in epithelial cells. Am J Physiol 1996;270(3 Pt 1):C794-802.

32. Di Lorenzo M, Bass J, Krantis A. Use of L-arginine in the treatment of experimental necrotizing enterocolitis. J Pediatr Surg 1995;30:235-40; discussion $240-1$.

33. Raul F, Galluser M, Schleiffer R, Gosse F, Hasselmann M, Seiler N. Beneficial effects of L-arginine on intestinal epithelial restitution after ischemic damage in rats. Digestion 1995;56:400-5.
34. Schleiffer R, Raul F. Prophylactic administration of L-arginine improves the intestinal barrier function after mesenteric ischaemia. Gut 1996;39:194-8.

35. Amin HJ, Zamora SA, McMillan DD, et al. Arginine supplementation prevents necrotizing enterocolitis in the premature infant. J Pediatr 2002;140:425-31.

36. Shah P, Shah V. Arginine supplementation for prevention of necrotising enterocolitis in preterm infants. Cochrane Database Syst Rev 2007;3:CD004339.

37. De Luis DA, Izaola O, Cuellar L, Terroba MC, Martin T, Ventosa M. A randomized double-blind clinical trial with two different doses of arginine enhanced enteral nutrition in postsurgical cancer patients. Eur Rev Med Pharmacol Sci 2010;14:941-5.

38. Wu G, Knabe DA. Arginine synthesis in enterocytes of neonatal pigs. Am J Physiol 1995;269(3 Pt 2):R621-9.

39. Adibi SA, Mercer DW. Protein digestion in human intestine as reflected in luminal, mucosal, and plasma amino acid concentrations after meals. J Clin Invest 1973;52:1586-94.

40. Claude P. Morphological factors influencing transepithelial permeability: a model for the resistance of the zonula occludens. J Membr Biol 1978;39:219-32.

41. Taylor CT, Dzus AL, Colgan SP. Autocrine regulation of epithelial permeability by hypoxia: role for polarized release of tumor necrosis factor alpha. Gastroenterology 1998;114:657-68. 